\title{
Considerations of education in the field of biophotonics in engineering: the experience of the subject Fundamentals of Biophotonics
}

\author{
F. Fanjul-Vélez*, J. L. Arce-Diego* \\ Applied Optical Techniques Group, TEISA Department, University of Cantabria \\ Av de los Castros s/n, 39005 Santander (Spain)
}

\begin{abstract}
Education in the field of photonics is usually somehow complex due to the fact that most of the programs include just a few subjects on the field, apart from specific Master programs in Photonics. There are also specific doctorate programs dealing with photonics. Apart from the problems shared with photonics in education in general, biophotonics specifically needs an interdisciplinary approach between biomedical and technical or scientific fields. In this work, we present our education experience in teaching the subject Fundamentals of Biophotonics, intended preferentially to engineering Bachelor and Master degrees students, but also to science and medicine students. First it was necessary to join a teaching group coming from the scientific technical and medical fields, working together in the subject. This task was easier as our research group, the Applied Optical Techniques group, had previous contacts and experience in working with medicine professors and medical doctors at hospitals. The orientation of the subject, intended for both technical and medical students, has to be carefully selected. All this information could be employed by other education institutions willing to implement studies on biomedical optics.
\end{abstract}

Keywords: biomedical optics, biophotonics, optics education, interdisciplinary teaching

\section{INTRODUCTION}

Photonics education is usually somehow complex due to the fact that most of the programs include just a few subjects on the field, apart from specific Master programs in Photonics. Typically, education in photonics is implemented by means of subjects in science or engineering Bachelor and Master levels, such as those at the Universities of Bochum, Michigan, Vandelbirt, Lund, Amsterdam or Arizona, for instance. There are also specific doctorate programs dealing with photonics.

In the particular field of biophotonics [1], education difficulties are even greater, as it is complex to find subjects on the field in bachelor or master programs, and even in doctoral programs. Apart from the problems shared with photonics in education in general, biophotonics specifically needs an interdisciplinary approach between biomedical and technical or scientific fields. This relationship is always needed, but most of the time is hard to establish or even to maintain.

In this work, we present our education experience in teaching the subject Fundamentals of Biophotonics, intended preferentially to engineering Bachelor and Master degrees students, but also to science and medicine students. First it was necessary to join a teaching group coming from the scientific technical and medical fields, working together in the subject. This task was easier as our research group, the Applied Optical Techniques group, had previous contacts and experience in working with medicine professors and medical doctors at hospitals. The orientation of the subject, intended for both technical and medical students, has to be carefully selected, in order for it to be as self-contained as possible. Professors' profiles, subject program, evaluation results and students' opinions are presented. All this information could be employed by other education institutions willing to implement studies on biomedical optics.

Section 2 contains the fundamentals of the teaching program and professorship involved. In Section 3 the essential of subject program is shown in detail. Section 4 presents some results, and Section 5 comprises the conclusions.

*fanjulf@unican.es; arcedj@unican.es; phone +34 942206730; www.teisa.unican.es/toa

Photonics, Devices, and Systems VII, edited by Karel Fliegel, Petr Páta,

Proc. of SPIE Vol. 10603, 106031D · (c) 2017 SPIE · CCC code:

0277-786X/17/\$18 - doi: $10.1117 / 12.2292445$

Proc. of SPIE Vol. 10603 106031D-1 


\section{TEACHING PROGRAM AND PROFESSORSHIP}

The subject teaching program is in accordance to the multidisciplinary approach the subject is intended for. As it is recommended for both engineers and physicists, as long as for medical or biomedical students, the program should be self-contained. The elaboration of the program involved looking at the programs of the Universities of Bochum, Michigan, Vandelbirt, Lund, Amsterdam or Arizona, among others. The proposed program appears below:

- $\quad$ 1. Nature of light and basic concepts

- 2. Basic optical devices in biomedical applications

- 3. Basic biological tissues types

- 4. Light propagation in biological tissues

- 5. Basic light-tissue interactions

- 6. Optical treatment techniques

- 7. Optical diagnostic techniques

- $\quad$ 8. Endoscopes and fiber probes

This program assures that students coming from the biomedical field receive appropriate background on optics and optical devices (in themes 1 and 2), and students on the technical side are aware of biological tissues fundamentals (theme 3). Of course, the approach of themes 6,7 and 8 must be broad enough in order to meet the expectations of both students' types.

Regarding professorship, it was decided to take advantage of medical professors in order to favor interdisciplinarity. Although engineering professors taught most of the themes, medicine professors taught theme 3 and parts of the clinical applications. This fact also encourages enrollment of medicine students.

\section{FUNDAMENTALS OF SUBJECT PROGRAM}

The teaching program involved several topics, part of which will be shown in this section. The selection of these topics was based on the comparison with other programs, as mentioned, and also on the fact that a general view of biomedical applications of optical radiation is given. Furthermore, a clinical perspective was given when selecting applications in the diagnosis, treatment and surgery fields.

\subsection{Biomedical optics advantages}

Biomedical optics deals with the application of optical radiation to biological tissues. In this sense, it is a particular case of the broader Biomedical Engineering field. Conventional techniques present several disadvantages, such as the use of ionizing radiation (X-rays, Nuclear Medicine), low resolution (X-rays), contact needed (ultrasound), expensive and slow procedure (MRI), invasive procedures (surgery), or non-desired side effects (chemotherapy), among others. Advantages of optical techniques in biomedicine when compared with conventional techniques appear both in treatment and diagnostic techniques. In diagnosis they provide non-invasive or minimally invasive character (by endoscopy), noncontact, non-ionizing and high resolution applications. In treatment, they show non-invasive or minimally invasive character by endoscopy), non-contact and great control and localization of the treatment area or volume. All these advantages are stressed in order to justify the relevance of optics in the medical field.

\subsection{Optical properties of biological tissues}

The knowledge of the optical properties plays an important role in diagnostic applications, therapy and surgery in biomedicine. Propagation of light in turbid media involves photophysical processes that affect the propagation of light. They can be quantified by several parameters: index of refraction, absorption, scattering (Rayleigh, Mie, geometric), reduced scattering coefficient, anisotropy of scattering, structural anisotropy, or even nonlinearities [2].

Absorption processes are relevant in diagnostic applications, as transitions between two energy levels of a molecule that are well defined at specific wavelengths could serve as a spectral fingerprint of the molecule for diagnostic purposes. In therapeutic applications, absorption of energy is the primary mechanism that allows light from a laser to produce physical effects on tissue for treatment purposes [3]. 
Scattering will occur when the guest material occupies only a localized region within the host material [4]. Scattering interactions are often dominant mechanisms affecting light propagation in biological tissues. Scattering processes are relevant in diagnostic applications, as scattering depends on the size, morphology, and structure of the components in tissues. Variations in these components due to disease would affect scattering properties, thus providing a means for diagnostic purposes, especially in imaging applications. For therapeutic applications, scattering signals can be used to determine optimal light dosimetry (e.g., during laser-based treatment procedures) and provide useful feedback during therapy.

There are also phenomena arising from the anisotropy [5, 6]: birefringence or unequal delay of two orthogonal polarization components, dichroism or unequal attenuation of two orthogonal polarization components, optical activity or rotation of the incident polarization state, and Faraday effect or rotation proportional to the applied external magnetic field. Inelastic scattering implies that the scattered photon either loses energy to (Stokes) or gains energy from (antiStokes) the molecule. Raman scattering makes the frequencies of scattered photons shift from the incident frequency by amounts that are characteristic of molecular transitions (molecular characterization of tissues). Brillouin scattering can also be used with that purpose.

\subsection{Biological tissues}

Biological tissues can be mainly characterized by their morphostructural characteristics, as they are of utmost relevance in biomedical optics [7]. Light-tissue interaction depends strongly on the morphostructure. The analysis method is usually histology. It reveals that biological tissues are usually heterogeneous and multilayered. Biological tissues can be hardly classified in the following categories: epithelial tissue, conective tissue, blood tissue, muscular tissue, and nervous tissue.

Epithelial tissue is composed by cells in adjacent layers, and they surround the body and internal organs. Cell types are plane or squamous, cubic, or cylindrical, with a varying number of layers, from simple or stratified epithelium. Conective tissue is usually disperse, highly heterogeneous, and contains cells with star form and oval nucleus. It forms collagen fibers, reticular and elastic fibers. Blood tissue contains intercellular fluid or plasma, and cells (erythrocytes, with a biconcave disc form, leucocytes, and platelets, with biconvex oval disc form). Muscular tissue is divided into skeletal muscle, with long fibers, cardiac muscle, with mononuclear or binuclear cells, and smooth muscle. Nervous tissue is formed by neurons and various types of glial cells or neuroglia. It involves the central nervous system (brain, cerebellum and spinal cord), and the peripheral nervous system (nerves, ganglia and nerve endings).

\subsection{Light-tissue interactions}

Light-tissue interactions comprise non-radiative light-matter interaction mechanisms. They originate the effects on biological tissues that allow treatment, characterization or surgery. They appear as a function of the radiation properties, and also on the biological tissues characteristics. The main mechanisms are photodisruption $\left(\sim 10^{13} \mathrm{~W} / \mathrm{cm}^{2}, \sim \mathrm{ps}, \mathrm{ns}\right)$, plasma-induced ablation $\left(\sim 10^{12} \mathrm{~W} / \mathrm{cm}^{2}, \sim \mathrm{ps}\right)$, photoablation $\left(\sim 10^{9} \mathrm{~W} / \mathrm{cm}^{2}, \sim \mathrm{ns}\right)$, thermal interaction $\left(\sim 10^{3} \mathrm{~W} / \mathrm{cm}^{2}, \sim \mathrm{ms}\right.$, $\mathrm{s})$, and photochemical interaction $\left(\sim 1 \mathrm{~W} / \mathrm{cm}^{2}, \sim \mathrm{s}\right.$, $\left.\mathrm{min}\right)$.

Photochemical interaction is employed in techniques such as Photodynamic Therapy [8], which is an optical treatment approach, photosensitizer-mediated, for tumoral tissue destruction. It is also the main mechanism in biostimulation that employs no photosensitizers, and is intended for a better prognosis of the pathology. Thermal interaction appears when there is a temperature increase in the irradiated biological tissue. Thermal interaction gives rise to several effects: vaporization, carbonization, coagulation, melting. As a result of the interaction several biological effects can occur: links destruction, changes in membranes, enzymatic activity reduction, inhibition of repairing cellular mechanisms, denaturalization of proteins and collagen, necrosis, mechanical rupture, tissue carbonization, or smoke. It is used in Thermotherapy [9], an optical treatment techniques with controlled temperature increase, or in interstitial thermotherapy, intended for tumor treatment by fiber-based hyperthermia. Photoablation is an interaction that provokes molecular links rupture in tissue, caused by high energy photons (UV). It is employed in laser surgery, precise cut with variable depth, or corneal refractive surgery. Plasma-induced ablation is an interaction provoked by the generation of plasma in the tissue. It produces a clean ablation, sometimes with plasma ejection. Applications comprise laser surgery [10], corneal refractive surgery, tooth decay treatment, or removal of dental plaque. Photodisruption generates mechanical forces and waves in the tissue, which provoke rupture in the material with matter ejection. It is mainly applied in the industry for materials cutting, or lenses fragmentation, and in medicine for low precision surgery, such as removal of kidney stones. 


\subsection{Optical treatment techniques}

As previously exposed, there are advantages of optical techniques compared to conventional techniques. These advantages are particularized in several treatment techniques, such as Photodynamic Therapy, Low level laser therapy, Thermotherapy, Hyperthermia or laser surgery.

In Photodynamic Therapy photochemical interaction is used. The cromophore is a photosensitive exogenous substance, with which the radiation interacts and causes it to react. The goal of treatment is malignant tissue destruction by controlled necrosis. It is intended usually in cancer tissues, and provides results of great interest. The procedure includes lesion location, inoculation of photosensitive substance which accumulates mainly in the malignant tissue, irradiation with optical source, photochemical interaction, photosensitizer reaction, generation of reactives and destructive effect on tissue [11].

Thermotherapy uses the thermal interaction. Optical radiation is transferred to the tissue and converted into thermal energy. As a consequence, the tissue temperature increases. The temperature increase can have beneficial effects. It is necessary to control the temperature increase, as heat damage must be avoided. For this reason thermal interaction modeling is needed. Heat transmission mechanisms are mainly conduction, convection and radiation. Thermal properties of tissues must be known. The bio-heat equation can cope with this issue [12]:

$$
\int_{V} q(\vec{r}, t) d V=\int_{V} \rho c \frac{d T(\vec{r}, t)}{d t} d V-\int_{S} k \nabla T(\vec{r}, t) \hat{n} d S-\int_{V} \rho_{b} c_{b} w_{b}\left[T_{a r t}(\vec{r}, t)-T_{\text {ven }}(\vec{r}, t)\right] d V
$$

Applications include neovascularization, transpupillary thermotherapy, intraocular tumors (choroid), macular degeneration (for choroidal neovascularization), and otolaryngology.

Laser surgery usually relies on photoablation or plasma-induced ablation [10]. The aim is to cut the tissue with precision in order to become the optical scalpel. Surgeons have then a precision tool. Applications are accurate tumor excision, neurosurgery, prostate surgery, corneal refractive surgery or even suture.

\subsection{Optical diagnostic techniques}

Diagnostic optical techniques include optical microscopy, optical tomography (coherent and diffuse), spectroscopy (IR, fluorescence, Raman), and even more advanced techniques [13], such as Optical Coherence Tomography [14], see Figure 1.

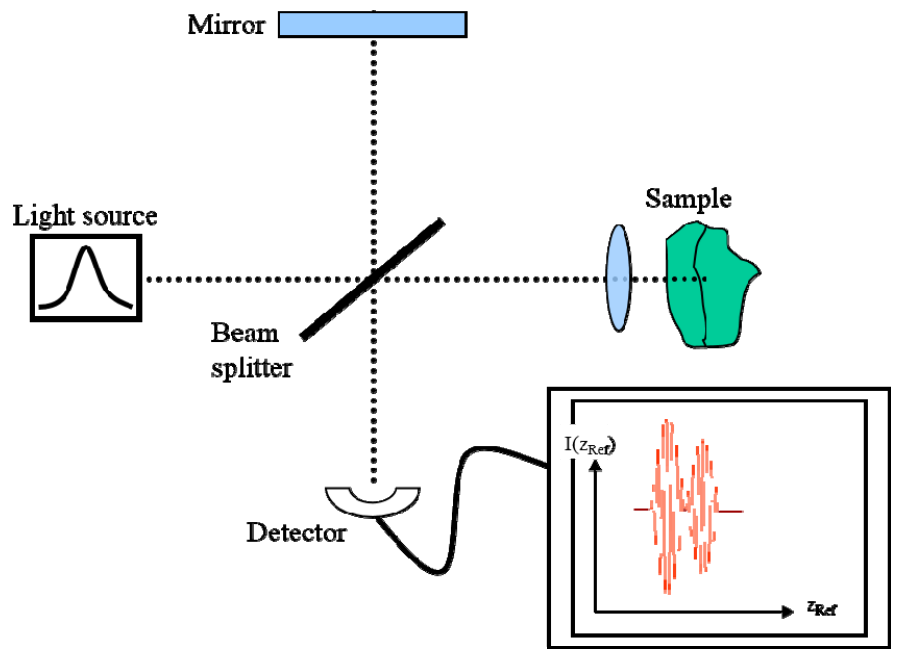

Figure 1. Basic scheme of Optical Coherence Tomography (Time Domain), based on a Michelson interferometer. 
Ultra high resolution OCT can provide even subcellular resolution, by reducing the coherence length of the source. This fact increments spectral width, as expressed in the next equation.

$$
l_{C}=c \tau_{C} \propto \lambda_{0}^{2} / \Delta \lambda
$$

Difficulties in practice include chromatic aberration, polarization mismatch between the arms of the interferometer, complex electronics used in detection, ultra wideband without affecting sensitivity, adaptive filters and scanning mechanical movement.

Different spectroscopic techniques are based on the types of light-tissue interactions: Raman Spectroscopy. Fluorescence Spectroscopy. Elastic Scattering Spectroscopy and Diffuse Refectance Spectroscopy. Even absorption Spectroscopy is employed.

Fluorescence techniques allow in vivo diagnosis without sample extraction [15]. Information is obtained in real time. Luminiscence Spectroscopy involves fluorescence and phosphorescence. They are based on optical detection and spectral analysis of the light emitted in a substance that suffers a transition from an excited electronic state to a lower one. Fluorescence diagnostic methods employ either endogenous chromophores in tissues, or exogenous fluorescent chromophores or their precursors (ALA) [16].

\subsection{Endoscopes and optical probes}

All the previously mentioned optical techniques can be made minimally invasive by the addition of endoscopes and optical fiber probes [17]. This fact has renewed the practice of medicine, when compared with conventional techniques. These approaches are employed in digestive endoscopy of the upper part, enteroscopy, colonoscopy, pancreatographic endoscopy, digestive tube endoscopy and surgery, pediatric endoscopy, histology and cytology, hemostasis, coagulation with argon laser, Photodynamic Therapy, polypectomy, sphincterotomy, mucosal resection and submucosa dissection, gastrostomy and jejunostomy, prosthesis, Zenker diverticulum, or gastroesophagic reflux.

Optical probes are designed for applications such as glucose level by diffuse reflection and Raman spectroscopy, gas concentration measurement in blood, breathing monitoring, $\mathrm{pH}$ in blood and stomach, pressure, perfusion by fluometry, laser Doppler, intraocular measurements by interferometry, skin characteristics, tumor diagnosis, or teeth [18]. More advances strategies use capsule endoscopy.

\subsection{Laboratory exercises}

All the program included laboratory exercises, as practice seems to be fundamental in this specific field. Laboratory exercises covered biological tissues morphostructure by histological microscopy, basics of optical radiation by measuring irradiance and spectra of several types of optical sources, basic spectroscopy on tissue-mimicking phantoms, basics of coherence and fiber optics propagation for endoscopy. Students from with biomedical background were much more confident with tissue morphostructure, while scientific technical students presented a better performance on optical parameters in general.

\section{RESULTS}

The articulation of such a multidisciplinary subject presented, as reported, several difficulties. Fortunately, the Applied Optical Techniques Group has already developed a research network with colleagues at the Faculty of Medicine and at the Marqués de Valdecilla University Hospital. This fact made all the process easier. Even terminology was a big issue when trying to coordinate the different themes of the subject. Once the teaching approach was clear and adequately coordinated, it was very important to be sure that the subject would be greatly self-contained. The background of the students was so diverse that this point was crucial.

An initial survey was made for the students to express their expectations on the subject. They were asked to evaluate the subject outline, and what they would like to be taught on, if something different from the program contents. The results show that practically all the students (more than 95\%) agree with the proposed subject outline, and they even consider it of high relevance $(90 \%)$. Some students write that the topics seem to be quite wide for a subject with such number of credits (around 10\%). Regarding expectations, most of the students coming from technical studies were just curious to know how they could give their knowledge a different application field (80\%). Some of the students even manifest that 
their initial intention was to carry a biological or medical degree (5\%), and they see this subject as an opportunity to regain that intention. Almost all the biomedical students expect the subject to clarify mechanisms or fundamental issues that they do not acquire in their degree, but are relevant as they are supposed to employ those techniques in clinical praxis (95\%).

Another survey was made after the course. In this one, the majority of the students considered following the subject a good idea, and a helpful view of the field $(90 \%)$. Some of them, particularly those of the biomedical field, had some difficulties in following some technical details, but this issue did not prevent them to follow the general subject (20\%). The evaluation results, based on a combination of a test of general concepts, and a written essay on some of the topics of the subject, was successful and remarked the interest of the students $(100 \%$ of the students passed, with a $20 \%$ of high marks over 8 out of 10).

\section{CONCLUSIONS}

Photonics education requires special care, as it is not usually widespread. This fact is even more pronounced when talking about biophotonics. Biophotonics education requires a multidisciplinary approach, comprising knowledge from engineering and physics, and on the biomedical side. This task was accomplished at our University by means of the subject Fundamentals of Biophotonics.

The implemented program and contents have been shown. The subject is thought to be self-contained, as students from quite different fields are expected to attend. General concepts about light, light propagation and biological tissues cope with this issue. Multidisciplinary professorship is involved, taking advantage of the previous research alliances of the Applied Optical Techniques group.

The results are quite encouraging, as the general opinion of the students is positive. Technological students find it quite interesting to know about a non-traditional application field. Biomedical students take advantage of the insight into the fundamentals of techniques that they are expected to use in medical praxis. The evaluation results further remark the general interest of the students.

\section{ACKNOWLEDGEMENTS}

This work has been partially supported by the project "New active phases in transition metals and rare earth nano-oxides stabilized at high pressure" (MAT2015-69508-P) of the Spanish Ministry of Economy and Competitiveness, cofunded by FEDER funds, and by the San Cándido Foundation.

\section{REFERENCES}

[1] Vo-Dinh, T., [Biomedical Photonics Handbook], CRC Press, Boca Raton (2015).

[2] Ormachea, O., Romanov, O. G., Tolstik, A. L., Arce Diego, J. L., Fanjul Vélez, F. and Pereda Cubián, D., "Frequency up-conversion of coherent images by intracavity nondegenerate four-wave mixing," Optics Express 14, 8298-8304 (2006).

[3] Salas-García, I., Fanjul-Vélez, F. and Arce-Diego, J. L., "Photosensitizer absorption coefficient modelling and necrosis prediction during photodynamic therapy," Journal of Photochemistry and Photobiology B: Biology 114, 79-86 (2012).

[4] Ortega-Quijano, N., Fanjul-Vélez, F. and Arce-Diego, J. L., "Physically meaningful depolarization metric based on the differential Mueller matrix," Optics Letters 40, 3280-3283 (2015).

[5] Ortega-Quijano, N., Fanjul-Vélez, F., de Cos-Pérez, J. and Arce-Diego, J. L., "Analysis of the depolarizing properties of normal and adenomatous polyps in colon mucosa for the early diagnosis of precancerous lesions," Optics Communications 284, $4852-4856$ (2011).

[6] Fanjul-Vélez, F., Ortega-Quijano, N. and Arce-Diego, J. L., "Polarimetry Group Theory analysis in biological tissue phantoms by Mueller Coherency matrix," Optics Communications 283, 4525-4530 (2010). 
[7] Salas-García, I., Fanjul-Vélez, F. and Arce-Diego, J. L., "Influence of the human skin tumor type in Photodynamic Therapy analysed by a predictive model," International Journal of Photoenergy 2012, 759205 (2012).

[8] Fanjul-Vélez, F., Salas-García, I., Fernández-Fernández, L. A., López-Escobar, M., Buelta-Carrillo, L., Ortega-Quijano, N. and Arce-Diego, J. L., "Photochemical model of Photodynamic Therapy applied to skin diseases by a topical photosensitizer," Proc. SPIE 7373, 73730S (2009).

[9] Fanjul-Vélez, F. and Arce-Diego, J. L., "Modeling thermotherapy in vocal cords novel laser endoscopic treatment," Lasers in Medical Science 23(2), 169-177 (2008).

[10]Fanjul-Vélez, F., Salas-García, I. and Arce-Diego, J. L., "Analysis of laser surgery in non-melanoma skin cancer for optimal tissue removal," Laser Physics 25, 025606-1, 8 (2015).

[11] Fanjul Vélez, F., Romanov, O. G., López-Escobar, M., Ortega-Quijano, N. and Arce Diego, J. L., "Necrosis prediction of Photodynamic Therapy applied to skin disorders," Proc. SPIE 7161, 71610P (2009).

[12] Fanjul-Vélez, F., Romanov, O. G. and Arce-Diego, J. L., "Efficient 3D numerical approach for temperature prediction in laser irradiated biological tissues," Computers in Biology and Medicine 39, 810-817 (2009).

[13] Fanjul-Vélez, F. and, Arce-Diego, J. L., "Polarimetry of birefringent biological tissues with arbitrary fibril orientation and variable incidence angle," Optics Letters 35, 1163-1165 (2010).

[14]Fanjul-Vélez, F., Pircher, M., Baumann, B., Götzinger, E., Hitzenberger, C. K. and Arce-Diego, J. L., "Polarimetric analysis of the human cornea by Polarization Sensitive Optical Coherence Tomography," Journal of Biomedical Optics 15, 056004 (2010).

[15] Salas-García, I., Fanjul-Vélez, F. and Arce-Diego, J. L., "Superficial radially-resolved fluorescence and threedimensional photochemical time-dependent model for Photodynamic Therapy," Optics Letters 39, 1845-1848 (2014).

[16] Salas-García, I., Fanjul-Vélez, F. and Arce-Diego, J. L., "Spatial photosensitizer fluorescence emission predictive analysis for photodynamic therapy monitoring applied to a skin disease," Optics Communications 285, 1581-1588 (2012).

[17] Ortega-Quijano, N., Arce-Diego, J. L. and Fanjul-Vélez, F., "Quality limiting factors of imaging endoscopes based on optical fiber bundles," Proc. SPIE 6991, 69910U (2008).

[18] Ortega-Quijano, N., Fanjul-Vélez, F. and Arce-Diego, J. L., "Optical crosstalk influence in fiber imaging endoscopes design,” Optics Communications 283, 633-638 (2010). 